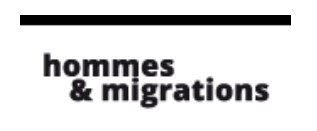

\section{Hommes \& migrations}

Revue française de référence sur les dynamiques

migratoires

$1320 \mid 2018$

Au prisme de la consommation

\title{
La mise en scène de l'authenticité
}

Stratégies de distinction de restaurants arabe et vietnamien dans le processus de gentrification de la ville de Berlin

\section{Miriam Stock et Antonie Schmiz}

Traducteur : Valentine Meunier

\section{CpenEdition}

\section{Journals}

Édition électronique

URL : http://journals.openedition.org/hommesmigrations/4054

DOI : 10.4000/hommesmigrations.4054

ISSN : 2262-3353

Éditeur

Musée national de l'histoire de l'immigration

Édition imprimée

Date de publication : 1 janvier 2018

Pagination : 69-77

ISBN : 978-2-919040-40-7

ISSN : 1142-852X

\section{Référence électronique}

Miriam Stock et Antonie Schmiz, «La mise en scène de l'authenticité », Hommes \& migrations [En

ligne], 1320 | 2018, mis en ligne le 01 janvier 2021, consulté le 06 janvier 2021. URL : http://

journals.openedition.org/hommesmigrations/4054 ; DOI : https://doi.org/10.4000/

hommesmigrations. 4054 


\title{
LA MISE EN SCENNE DE L'AUTHENTICITÉ : STRATÉGIES DE DISTINCTION DE RESTAURANTS ARABES ET VIETNAMIENS DANS LE PROCESSUS DE GENTRIFICATION DE LA VILLE DE BERLIN
}

Par MIRIAM STOCK, professeur junior en études culturelles, École supérieure de pédagogie de Schwäbisch Gmünd, et ANTONIE SCHMIZ, professeure junior en géographie des migrations, Institut d'études migratoires et interculturelles (IMIS), université de Osnabruck.

\begin{abstract}
À Berlin, les expériences gastronomiques « authentiques » se multiplient dans les quartiers du centre-ville en cours de gentrification. Des falafels orientaux à la cuisine vietnamienne, I'aménagement et la carte de ces restaurants évoluent afin de répondre aux goûts des Berlinois. Jouant avec les représentations exotiques dont les commerces ethniques sont l'objet, cette offre culinaire s'adresse à une clientèle cosmopolite et urbaine, à la recherche d'une cuisine saine et ouverte sur le monde. Ainsi, de nouvelles formes de restauration voient le jour pour proposer une expérience culinaire qui tranche avec les traditionnelles mises en scène folkloriques.
\end{abstract}

\footnotetext{
"Lorsque les nuages noirs obscurcissent le ciel, la balade au marché aux puces du Mauerpark s'achève par un repas spontané au Phönizier. Dehors tombent les premières gouttes, à l'intérieur les clients s'installent douillettement sur une estrade pourvue de coussins moelleux. (...) Lorsqu'on demande au chef cuisinier quelles épices parfument le plat, il répond par un sourire énigmatique et renvoie à la longue tradition et à la richesse de la cuisine libanaise ${ }^{1}$. ”

"Lorsqu'après avoir remonté la Pannierstraße, grise et détrempée par la pluie, on arrive à la toute jeune Maison Han à Neukölln, le blues d'automne s'envole. (...) Mais le véritable point d'orgue c'est le petitdéjeuner vietnamien - car si on s'est déjà nourri de quantité de Bánh mì ou autre Báhn Bao au déjeuner ou au dîner, on ignore tout, chez nous, de la culture vietnamienne du petit-déjeuner². »
}

À Berlin, les restaurants "ethniques » font partie intégrante de la vie des quartiers qui sont traversés par des processus de gentrification. C'est ce que laissent entendre les citations reproduites ci-dessus décrivant les expériences gastronomiques de deux critiques culinaires dans deux restaurants - l'un situé dans le quartier de Prenzlauer Berg en 2006 et l'autre au nord de Neukölln : la fabrication de l'authenticité joue un rôle essentiel dans la négociation entre consommation et mise en scène d'une 
économie « ethnique ». Partant de cette hypothèse, il s'agit de comprendre comment les entrepreneurs migrants donnent à voir différentes formes d'authenticité dans leurs restaurants labellisés « ethniques » dans une ville comme Berlin.

\section{Économies migrantes, gentrification et authenticité}

Plusieurs grandes villes allemandes ont récemment découvert que la diversité ethnoculturelle, qui laisse son empreinte sur de vastes pans de l'économie migrante, pouvait être une ressource. Alors que les Chinatowns et Little Italies de Paris, New York et Toronto contribuent depuis de nombreuses années à l'image positive de ces villes et sont en quelque sorte vendues comme des attractions touristiques, certains secteurs de l'économie migrante ont dernièrement été incorporés dans le marketing urbain, ou city branding, dans les métropoles allemandes ${ }^{3}$. La situation spatiale des restaurants ethniques dans les quartiers des centres-villes nous paraît particulièrement pertinente, car ces lieux s'adressent à une clientèle jeune, qualifiée, mobile et créative, que les métropoles tentent actuellement de s'attacher à l'échelle mondiale ${ }^{4}$.

On observe tout particulièrement cette corrélation à Berlin, "métropole de la culture underground ${ }^{5}$ ". C'est notamment un multiculturalisme consumériste proposé par l'économie migrante qui a attiré les jeunes « talents » dans la ville et continue de le faire. Les entreprises du secteur culturel et de la création (Kreativunternehmen) ainsi que les start-ups sont devenues l'un des principaux facteurs de la croissance économique de cette ville en rapide mutation ${ }^{6}$.

La mise en scène d'une authenticité de la consommation figure au cœur de ce processus, ainsi que l'a établi Sharon Zukin dans son livre Naked City. The live and death of authentic places ${ }^{7}$. Dans une société fortement mondialisée et hybride, la production de l'authenticité ne se fonde pas sur une origine véritable, mais plutôt sur l'idée de rendre tangible certaines origines imaginaires à l'ère de la modernité tardive. Ainsi, selon Sharon Zukin, l'authenticité ne peut être vécue que de l'extérieur8.

L'explosion et le succès mondial de la commercialisation de l'authenticité ne doivent ici rien au hasard. Ils reposent sur des transformations socioéconomiques radicales à l'ère du capitalisme tardif, dans lequel la culture figure désormais au cœur de l'exploitation économique. Sharon Zukin parle à ce propos d'une «économie symbolique ${ }^{9}$. Sighard Neckel qualifie pour sa part cette nouvelle forme d'économie de marché de "capitalisme culture ${ }^{10}$ ". " "L'authenticité" comme valorisation culturelle devient ce faisant une monnaie d'échange importante ${ }^{11}$ ".

Les pionniers, très étudiés, de ce capitalisme culturel sont les membres d'une nouvelle classe moyenne blanche cultivée ${ }^{12}$. Dans son ouvrage La Distinction, Pierre Bourdieu identifiait déjà cette classe comme une frange marginale des couches moyennes et la qualifiait de "nouvelle petite bourgeoisie $e^{13}$ ".

3. Volkan Aytar, Jan Rath, "Introduction: Ethnic neighborhoods as places of leisure and consumption ", in Volkan Aytar, Jan Rath (dir.), Selling Ethnic Neighborhoods, Londres, New York, Routledge, 2012 , pp. 1-15; Keith Dinnie, City Branding, Basingstoke, Palgrave Macmillan, 2010 ; Stephanie Hemelryk Donald, Eleonore Kofman, Catherine Kevin, "Introduction: Processes of cosmopolitanism and parochialism ", in Stephanie Hemelryk Donald, Eleonore Kofman, Catherine Kevin (dir.), Branding Cities, New York, Routledge, 2008, pp. 1-13; Antonie Schmiz, "Staging a "Chinatown" in Berlin. The role of city branding in the urban governance of ethnic diversity ", in European Urban and Regional Studies, vol. 24, $n^{\circ}$ 3, 2017. 4. Ans Rekers, Ronald van Kempen, "Location matters: Ethnic entrepreneurs and the spatial context ", in Jan Rath (dir.), Immigrant Businesses: The Economic, Political and Social Environment, Londres, Palgrave Macmillan, 2000, p. 63. 5. Stephan Lanz, Berlin aufgemischt. Abendländisch-Multikulturell-Kosmopolitisch? Die politische Konstruktion einer Einwanderungsstadt, Bielefeld, Transcript Verlag, 2007, p. 188. 6. Stephan Krätke, "City of Talents? Berlin's regional economy, socio-spatial fabric and "worst practice" urban governance ", in International Journal of Urban and Regional Research, vol. 28, n 3, 2004, p. 511-529. 7. Sharon Zukin, The Death and Life of Authentic Urban Places, New York/Oxford, OUP USA, 2010. 8. Sharon Zukin, " Consuming Authenticity ", in Cultural Studies, vol. 22, n5, 2008, p. 727. 9. Sharon Zukin, Cultures of Cities, Cambridge, Wiley-Blackwell, 1995. 10. Sighard Neckel, "Die Marktgesellschaft als kultureller Kapitalismus. Zum neuen Synkretismus von Ökonomie und Lebensform ", in Kurt Imhof, Thomas Eberle (dir.), Triumph und Elend des Neoliberalismus, Zurich, Seismo Verlag 2005, pp. 198-211.

11. Miriam Stock, Der Geschmack der Gentrifizierung : Arabische Imbisse in Berlin, Bielefeld, Transcript Verlag, 2013, pp. 253 s5. 12. Tim Butler, Gentrification and the Middle Classes, Hants, Ashgate Publishing Limited, 1997 ; "Living in a bubble: Gentrification and its "others" in north London ", in Urban Studies, vol. 40, n 12, 2003, pp. 2469-2486. 13. Pierre Bourdieu, La Distinction. Critique sociale du jugement, Paris, Les Éditions de Minuit, 1979. 
Composée entre autres d'artistes, de journalistes, de chercheurs ou de designers, elle a connu une forte croissance entre les années 1960 et 2010, en parallèle d'une augmentation des diplômés, de la tertiarisation de l'économie ainsi que d'une précarisation néolibérale. Elle est ainsi devenue une catégorie importante du monde professionnel et du travail contemporain ${ }^{14}$. Hautement qualifiés, les membres de cette classe se trouvent toutefois souvent dans une situation économique précaire. C'est grâce à leur capital culturel qu'ils s'assurent un statut social ${ }^{15}$, ce dernier se manifestant par une sensibilité à l'authentique, qui sert en quelque sorte de vecteur de distinction vis-à-vis des autres groupes sociaux.

Dans l'espace urbain, la «nouvelle petite bourgeoisie » s'affiche en particulier dans les secteurs en gentrification et s'est appropriée la consommation dans des quartiers entiers - dès les prémisses de leur valorisation. Il a déjà été scientifiquement établi que la marchandisation de l'authenticité comme vecteur de distinction joue un rôle très important, notamment dans les processus de gentrification ${ }^{16}$, que ce soit dans la demande en immeubles d'habitation anciens ou dans la fréquentation d'un restaurant « ethnique ». Pour reprendre les mots de Bourdieu ${ }^{17}$, la gentrification peut par conséquent être aussi analysée comme un processus par lequel le capital culturel est converti en plus-value économique.

On s'est, en revanche, beaucoup moins intéressé au capital culturel des entreprises de migrants qui ouvrent de nouvelles enseignes pendant la gentrification. Ils contribuent à définir le goût de la classe moyenne et rendent les quartiers plus attractifs aux yeux de ses membres. Les chercheurs "ethnicisent », en effet, souvent les migrants et analysent leurs pratiques entrepreneuriales comme l'expression d'une « origine " présumée. Ils n'interrogent pas davantage cette hypothèse. Pourtant, il serait également possible de les penser à la fois comme des pratiques de distinction inventives et comme des sources particulièrement efficaces d'accroissement des ventes ${ }^{18}$. Or, les travaux de Miriam Stock sur les falafels et la gentrification à Berlin ${ }^{19}$, à la base de cet article, ainsi que les recherches d'Erol Yildiz sur la gentrification dans un quartier de Cologne ${ }^{20}$ attestent du rôle central que peuvent jouer les entreprises de migrants dans le processus de valorisation urbaine.

Nous mettrons ici en lumière les stratégies de ces entrepreneurs durant différentes phases de la gentrification berlinoise en nous appuyant sur l'étude de deux propositions de restauration, l'une arabe, l'autre vietnamienne. Nous étudierons comment ces deux entreprises s'insèrent dans l'évolution du quartier et le regard qu'elles portent sur les produits « authentiques » qu'elles commercialisent.

\section{Der Phönizier, le snack oriental}

Der Phönizier a ouvert en $2004^{21}$ aux abords du Mauerpark, dans le quartier de Prenzlauer Berg, et vend des "spécialités libanaises ». Comme le montre la photo 1 , un tableau, accroché sur la façade extérieure recouverte de vigne vierge, présente une carte des menus, écrite à la main. Ce sont avant tout des plats typiques aux yeux des Berlinois - «falafel », «shawarma » et «halloumi »- qui sont proposés en sandwich ou à l'assiette. Une fois le seuil de la porte passé, on se retrouve devant une vitrine qui contient, rangés les uns à côté des autres, houmous, halloumi et salades dans des bols.

14. David Ley, The New Middle Classes and the Remaking of the Central City, Oxford, Oxford University Press 1996 ; Tim Butler, op. cit. 15. Pierre Bourdieu, op. cit. 16. Voir, par exemple, Michael Jager, "Class definition and the aesthetics of gentrification: Victoriana in Melbourne ", in Neil Smith, Peter Williams (dir.), Gentrification of the City, New York, Routledge, 1986, p. 78-91; David Ley, op. cit.; Matthew Rofe, "I want to be global": Theorising the gentrifying class as an emergent elite global community ", in Urban Studies, vol. 40, $n^{\circ}$ 12, 2003, pp. 2511-2526 ; Sharon Zukin, "Consuming Authenticity ", op. cit.; Sharon Zukin, The Death and Life of Authentic Urban Places, op. cit. 17. Pierre Bourdieu, op. cit. 18. Miriam Stock, op. cit., pp. 116 ss. 19. Ibid. 20. Erol Yildiz, "Migration und Diversität als urbane Ressource ", in Heike Herrmann, Carsten Keller, Rainer Neef, Renate Ruhne (dir.), Die Besonderheit des Städtischen: Entwicklungslinien der Stadt (Soziologie), Wiesbaden, Springer, 2011, pp. 125-143; Die weltoffene Stadt: Wie Migration Globalisierung zum urbanen Alltag macht, Bielefeld, Transcript Verlag, 2013. 21. Le restaurant a aujourd'hui (avril 2017) fermé définitivement ses portes (NDT). 
Photo 1. Der Phönizier, vue d'extérieur, Berlin, 2009.

Photo Miriam Stock. (๐ MIRIAM STOCK.

Derrière le comptoir tourne une broche, sur laquelle sont prélevés les morceaux de viande pour les shawarmas. Au fond, dans le coin, la poêle à frire est prête à cuire les falafels à la demande. Un autre espace qui surprend dès l'entrée est le coin pour s'asseoir à l'orientale. Il est situé dans la pièce de devant et est décoré de tapis à motifs, de coussins imprimés dans des tons chauds rouge et violet ainsi que de tables basses en bois. Il faut se déchausser avant de s'y installer.
À l'époque de l'enquête empirique en 2009-2010, le Phönizier est l'un des 17 falafels de Prenzlauer Berg $^{22}$, quartier bourgeois de Berlin-Est à faible population arabe. Contrairement à ce qui est souvent véhiculé dans l'opinion publique, les restaurants de falafels tels que le Phönizier n'ont jamais été à Berlin les bras commerciaux d'une communauté ethnique souhaitant rester entre soi. La plupart des propriétaires de ces commerces sont certes originaires de pays arabes ${ }^{23}$, mais ils ont ciblé dès le 
dans ce quartier [ils restent] en moyenne, un an et demi. Ils changent de domicile. Il y a toujours un changement de génération. Oui, on a déjà noté, les gens viennent une ou deux années et déménagent. Dans la ville ou en dehors, dans d'autres villes. " (Entretien du 6 mai 2009.)

À l'instar d'autres propriétaires de restaurants de falafels, il a adapté sa carte au public du quartier. Ainsi, le propriétaire met en scène ses plats comme des plats végétariens ou véganes. Il garnit ses généreux sandwiches de diverses salades et d'un mélange de sauce au yaourt et à la mangue ainsi que de sauce épicée. Une recette qui n'a pas Der Phönizier, coin pour s'asseoir, Berlin, 2009 Photo Miriam Stock. (c) MIRIAM STOCK. grand-chose à voir avec les modestes falafels de Beyrouth, affirme le propriétaire. Là-bas, un sandwich falafel coûte à peine la moitié d'un shawarma au poulet. La sauce à la mangue n'existe départ une clientèle jeune, issue des couches moyennes allemandes et européennes dans les quartiers en gentrification de Berlin. Ce mouvement a débuté à Schöneberg et Kreuzberg dans les années 1980, avant de gagner d'autres quartiers tels que Mitte, Prenzlauer Berg et Friedrichshain après la chute du Mur. Plus récemment, ce processus se poursuit à Neukölln. Ainsi, les enseignes de falafels faisaient et font partie intégrante de la gentrification et de la transformation urbaine ${ }^{24}$.

Le propriétaire du Phönizier est né au Liban. Il vit depuis les années 1990 en Allemagne et s'investit en parallèle dans le secteur de la construction. Il décrit sa clientèle comme composée de "jeunes gens » de 20 à 30 ans, ou 40 ans au maximum. La " scène » du quartier ne serait pas particulièrement riche, mais serait plus « culinaire » qu'ailleurs dans Berlin, où l'on consommerait principalement des saucisses au curry ou d'autres variétés de saucisses allemandes. Ses propos renvoient clairement au capital culturel de sa clientèle, lié non seulement à un niveau d'étude élevé, mais également à un goût incorporé distinctif ${ }^{25}$. Mais il relève aussi que cette même clientèle est très fluctuante - un trait typique de la gentrification berlinoise: "Ici, les clients ne sont pas des clients réguliers. C'est-à-dire que les clients pas, et les falafels sont davantage perçus comme un plat gras de pauvres que comme une nourriture saine. À Berlin, à l'inverse, et particulièrement dans son restaurant, les clients seraient très soucieux de la qualité et de la fraîcheur des produits. On voit ici clairement comment le falafel, en parallèle du processus de gentrification, a connu une revalorisation et un embourgeoisement.

Outre l'étiquette « libanaise », l'authentification des plats dans son restaurant s'effectue principalement par le biais de la décoration et de l'ambiance. Le coin oriental pour s'asseoir suscite effectivement des réactions positives parmi nos interlocuteurs, qui le jugent « authentique ». Ben, étudiant Erasmus de 24 ans originaire de Suisse, déclare : « Je trouve que ça a un air naturel avec les chichas [narguilés] et les coussins. C'est ainsi que je m'imagine [là-bas]. » Sarah, étudiante en musique de 22 ans, dit : « Oui, les coussins, ça donne un air confortable, on pourrait s'y asseoir et on a le sentiment de ne pas être à Berlin. » Elle ajoute qu'elle ne pense pas que le restaurant soit très fidèle à la réalité : "Je pense qu'au Liban ou ailleurs, il ne serait pas aussi chic et coquet. " Les consommateurs reproduisent dans leurs propos des stéréotypes socialement ancrés d'un Orient qu’Edward Saïd a déjà révélés dans son ouvrage 
majeur L'Orientalisme ${ }^{26}$ et qui sont également exploités, au moins en partie, au Phönizier. Sarah, qui n’a jamais été au Liban, fonde ses appréciations exclusivement sur des clichés du monde arabe véhiculés par les médias et la culture populaire, ainsi que sur sa perception des migrants arabes à Berlin ${ }^{27}$.

\section{Le pouvoir commercial des représentations folkloriques}

Le propriétaire du Phönizier souhaite précisément se démarquer de ces clichés exotiques avec son restaurant, même si cette intention reste subtile. Il poursuit, en effet, une stratégie commerciale de présentation orientale, mais en forçant sciemment le trait, entre kitsch et chic. Il dit lui-même à propos de la décoration : "C'était juste une idée que j'avais en tête, une rêverie. " (Entretien du 6 mai 2009.) Il a d'ailleurs acheté l'essentiel des coussins ornementés, narguilés et lampes décoratives dans des magasins berlinois.

Son jeu entre différents univers culturels de référence ne relève certainement pas du hasard, car il ne se sent pas particulièrement « arabe ». Il raconte ainsi au sujet du nom du restaurant : « Le Phönizier, oui, le terme vient de ce coin de la planète, ça a rapport avec le Liban. Beaucoup de Libanais se sentent avant tout Libanais. D'autres écrivent Al Arabi [l'Arabe]. Euxmêmes prennent un nom qui leur convient. » (Ibid.) Au lieu de recourir à l'écriture arabe souvent privilégiée par les restaurants de falafels, il utilise une police rappelant les hiéroglyphes et qui renvoie à la culture phénicienne, évitant ainsi toute connotation arabe. De plus, il vend différentes sortes de vins et de bières dans sa boutique - une exception dans ce genre de restaurants. Le propriétaire est un chrétien maronite. Au Liban, ce sont principalement les Maronites qui revendiquent des origines phéniciennes, notamment pour souligner leur longue histoire et ancrer leur distinction par rapport aux
Arabes musulmans. En outre, il porte un regard très critique sur la communauté arabe, majoritairement musulmane, des quartiers de Neukölln et de Wedding, qu'il juge très conservatrice. À l'inverse de Paris, où les chrétiens du Liban sont très nombreux, ce sont avant tout des populations musulmanes et pauvres qui se sont installées à Berlin-Ouest. Après avoir fui la guerre civile dans les années 1980, ils ont transité par Berlin-Est, seule porte qui leur était ouverte en Europe ${ }^{28}$.

Il a, cependant, parfaitement conscience que les goûts de ses clients penchent vers une forme d'orientalisme. Il est régulièrement restreint dans ses choix par leurs attentes. Il raconte, par exemple, que les clients se plaignent lorsqu'il passe de la musique douce occidentale et qu'ils réclament de la musique orientale (entretien du 6 mai 2009).

Le Phönizier fait partie de la catégorie des restaurants ethniques dans laquelle on insiste fortement sur le folklorique, ce qui est également souhaité par les clients. Les falafels orientalisés, à l'instar d'autres restaurants folklorisés, ont connu un important succès dans les premières phases de la gentrification berlinoise des années 1990 et $2000^{29}$. Depuis, de nouvelles mises en scène authentiques de restaurants ethniques s'imposent sur le marché berlinois, ainsi qu'en atteste le second exemple, un restaurant vietnamien spécialisé dans les petits-déjeuners.

\section{Maison Han, Breakfast vietnamien}

Maison Han est un restaurant de petits-déjeuners vietnamiens qui a ouvert ses portes en 2016 dans le nord du quartier de Neukölln. Il associe, dans ses locaux, gastronomie et torréfaction de café. Une fois la porte d'entrée franchie, les clients pénètrent dans une salle claire à grandes baies vitrées. Leur regard est attiré par un comptoir en bois très 
travail partagé (coworking). Associée au comptoir et aux tabourets de bar, cela crée le long de la fenêtre une ambiance minimaliste de travail et d'échange : des jeunes gens sont assis avec leurs ordinateurs et portables, faisant oublier de prime abord le caractère vietnamien du lieu.

Au début des années 1990, la gastronomie vietnamienne a, en effet, représenté la voie vers l'autonomie pour d'anciens employés contractuels issus de la communauté asiatique. Ils se sont mis à leur compte dans des roulottes ou des petits points de vente à emporter. À l'époque, on ne trouvait à BerlinEst que des spécialités panasiatiques, telles que les nouilles sautées (Chinapfanne) ou le canard frit ${ }^{30}$. La diversification des spécialités culinaires a débuté avec la reprise et l'ouverture de restaurants thaïs, chinois, coréens ou de sushis par des Vietnamiens qui puisaient

Maison Han, vue d'intérieur, Berlin, 2017. Photo Antonie Schmiz. (c) ANTONIE SCHMIZ.

présent derrière lequel on devine un percolateur, diverses vitrines et étagères présentant du café vietnamien fait maison, du chocolat et des gâteaux. Le personnel allemand et vietnamien sert la clientèle qui consomme des plats vietnamiens et des spécialités de café sur des meubles de style disparate. La nourriture est servie sur des plateaux dorés ou argentés dans des corbeilles traditionnelles en rotin, incarnant une esthétique de cantine moderne, mais ethnicisée. Ceci se démarque donc de la vaisselle en porcelaine aux motifs asymétriques choisie par la plupart des restaurants vietnamiens. Un pochoir réalisé directement sur les murs, au crépi parfois abîmé, indique les toilettes pour hommes (Monsieur) et femmes (Madame) en français. Une grande table fait office d'espace de ainsi dans différentes cuisines nationales sans révéler leur nationalité vietnamienne. Une phase que Pipo Bui qualifie de "partial masking ${ }^{31}$ » dans son étude sur la gastronomie vietnamienne à Berlin.

Au tournant du nouveau millénaire s'amorce une différenciation : de nouveaux restaurants font leur apparition dans le quartier de Mitte - à l'instar de Monsieur Vuong qui a ouvert ses portes en 2002 et proposent des soupes de nouilles et des plats de riz vietnamiens. Cette formule, revendiquant un ancrage "vietnamien " se répand rapidement dans les quartiers en cours de gentrification. Actuellement, c'est principalement dans le quartier très prisé du nord de Neukölln qu'apparaissent de nouvelles enseignes et restaurants vietnamiens exclusivement végans qui diffusent à l'échelle locale les grandes tendances culinaires actuelles. Cette distinction des établissements s'accompagne d'une mise en scène de leur cuisine comme authentiquement «vietnamienne ». S'inscrivant dans ce 
courant, la Maison Han démontre aussi que la cuisine vietnamienne, avec ses temps brefs de préparation dans de nouveaux espaces minimalistes, correspond parfaitement à une clientèle cosmopolite, urbaine et prisant autant la culture qu'une alimentation saine.

Le gérant de la Maison Han est, par ailleurs, un restaurateur expérimenté : il possède plusieurs établissements en Allemagne et notamment à Berlin. S'il se considère lui-même comme berlinois, ses parents sont des boat people vietnamiens réfugiés à BerlinOuest. De même, sa femme, qui exploite l'enseigne avec lui, fait partie de la seconde génération d'immigration vietnamienne: ses parents étant arrivés en Allemagne de l'Est (ex-République démocratique d'Allemagne) comme ouvriers contractuels. Il a étudié le management des médias et de la musique. Après son diplôme, il a commencé par concevoir et traduire des cartes de restaurant ; puis il a travaillé en cuisine, avant d'investir des parts dans des sociétés et d'établir ses propres établissements vietnamiens. Fort d'une formation de barista suivie au Salvador, le propriétaire de la Maison Han a ainsi créé sa propre marque: il importe, dans le cadre du commerce équitable, des grains d'arabica cultivés par de petits paysans des hauts plateaux vietnamiens, avant de les torréfier dans ses locaux, sans additifs ni exhausteurs de goût ou arômes artificiels.

Il recrute ses cuisiniers par l'affichage d'annonces dans le centre de commerce en gros Dong Xuan Center à Berlin-Lichtenberg. Ce sont moins des cuisiniers dotés d'une formation sanctionnée par un diplôme qui l'intéressent que ceux qui, comme lui, connaissent la cuisine vietnamienne par transmission familiale intergénérationnelle. Le gastronome adapte la cuisine traditionnelle à sa clientèle berlinoise et la vend comme une cuisine vietnamienne saine et moderne: "Dans ma cuisine en fait, je prépare un plat aussi authentique que possible, du point de vue de la couleur, de la consistance, à tous les points de vue. Et si j'y ajoute mon grain de sel, c'est parce que nous sommes la génération qui raffine un peu les choses. Mais ce n'est qu'une fois qu'on a réussi un plat traditionnel qu'on le modifie. Par exemple, si nous cuisinons un plat mijoté, au Vietnam on prendra les bas morceaux du boeuf, donc très gras. C'est certes délicieux, mais nous avons besoin de viande qui fonde plus facilement, moins grasse - aussi parce que c'est plus sain. Mais il faut toujours laisser de la graisse, parce que c'est authentique d'en laisser. " (Entretien du 29 octobre 2016.)

\section{La fabrique de l'authenticité}

Ici, l'entrepreneur fait lui-même fonction de représentant de l'extérieur, qui fabrique, pour ses clients, l'authenticité comme reconstruction d'un passé perdu $^{32}$. Ses stratégies entrepreneuriales permettent de retracer plusieurs tendances de la restauration vietnamienne: diversification grandissante, spécialisation et production de l'authenticité.

La mise en scène de ce qui est vietnamien passe avant tout par la cuisine : il propose des spécialités vietnamiennes épicées et des desserts combinés à des cafés raffinés. Sur demande, ses cafés peuvent également être préparés avec le filtre à café vietnamien "phin». Cette combinaison d'une présentation soignée, du choix de noms et de parfums, ainsi que de l'équipe de cuisiniers vietnamiens, participe de la mise en scène d'un restaurant de petitsdéjeuners vietnamiens authentique. Le propriétaire a, "pour une fois, choisi un nom qui ne dit pas clairement ses origines ». Le mot «Han » possède en effet des significations différentes en coréen, chinois, vietnamien et thaï, et est donc présent « pratiquement dans toute l'Asie » (entretien du 29 octobre 2016).

L'entrepreneur a délibérément opté pour un concept de salle moderne et minimaliste qu'il décrit comme suit sur son site Internet : "L'alliance du purisme industriel et de la matière brute ${ }^{33}$. »Il exprime ici clairement sa prise de distance avec les scénographies à caractère folkloriste des années 1990, car son concept spatial est « un projet au pôle opposé de la décoration et des ornementations exubérantes usuelles des restaurants asiatiques » (Ibid.). 
La Maison Han incarne ainsi une tentative de différenciation très actuelle que l'on repère dans la gastronomie dans son ensemble. La grande table de travail partagé permet d'allier lieu de travail, d'échanges et de déjeuner en commun. La mise en récit de l'espace par le propriétaire fait appel à des souvenirs de déjeuners entre amis ou avec sa famille nombreuse sur des tables de cette taille. Ce qui lui permet ici aussi de fabriquer de l'authenticité en recourant au contexte d'origine. La labellisation de certains plats comme " végans » s'inscrit également pleinement dans les tendances alimentaires du moment. Son restaurant de petits-déjeuners qui propose simultanément une nouvelle approche du café, filtré à la main, issu de grains lentement torréfiés et du commerce équitable, constitue un positionnement bien spécifique. La proximité entre restauration et production - ici la torréfaction - est aussi en vogue actuellement dans la gastronomie. L'entrepreneur distille subtilement des détails ethniques : l'inscription « Dalat », nom de la principale région de culture du café sur les hauts-plateaux vietnamiens, figure par exemple sur les sacs de café entreposés dans la boutique. On met en lien ici les préférences gustatives des clients avec leurs représentations de l'authenticité, en misant sur leur désir de distinction ${ }^{34}$.

\section{Conclusion}

Les deux enquêtes de terrain montrent quavec leurs restaurants labellisés " ethniques ", les entrepreneurs migrants jouent un rôle particulièrement actif dans la définition du goût à l'heure de la gentrification. Il en ressort tout aussi clairement que ces restaurants ne cherchent pas à proposer une offre empruntée à un contexte original, et reproduite avec la plus grande fidélité possible. Au contraire, l'enjeu consiste à mettre en scène un concept hybride, qui associe des éléments du pays d'origine, mais est adaptée aux tendances locales et aux préférences gustatives des Berlinois. Le propriétaire de la Maison Han déclare d'ailleurs : «Berlin nous a inspirés, aujourd'hui nous inspirons Berlin ${ }^{35}$ ". Ainsi, il ne faut pas évaluer ces concepts de restaurants selon des critères d'authenticité. La mise en scène de l'authenticité par le biais de l'espace et des produits devient une stratégie commerciale par laquelle les entrepreneurs investissent leur capital culturel.

Ces choix ne doivent rien au hasard. Ils reflètent divers ancrages, que ce soit du point de vue de la représentation « ethnique » ou de la gentrification. Tenant compte de sa clientèle jeune et diplômée du quartier de Prenzlauer Berg des années 2000, le Phönizier opte pour une scénographie à caractère folklorique dont il se démarque subtilement. Au contraire, la Maison Han manifeste sa distinction à l'égard de tout folklorisme asiatique grâce à une présentation moderne et minimaliste, qui interpelle le public international de l'économie créative fréquentant le quartier de Neukölln.

Le Phönizier incarne ainsi une phase de la transformation urbaine de Berlin pendant laquelle le capital culturel formait le cœur du vecteur distinctif. Berlin s'est ainsi longtemps vendue comme "pauvre mais sexy» («arm aber sexy»). De son côté, la Maison Han est caractéristique de la phase actuelle de revalorisation de quartiers berlinois, sous l'effet de l'installation d'une classe créative, de l'augmentation du nombre de start-ups et, par conséquent, de l'arrivée de nouvelles clientèles. Cette évolution se déroule en parallèle d'un marché immobilier de plus en plus attractif. Capital culturel et valorisation économique tendent à se confondre dans cette phase du développement de la ville tandis que de nouveaux paysages consuméristes redéfinissent les formes d'inclusion et d'exclusion à l'œuvre. 